\title{
II
}

\section{Current stellar dynamo theory}

- The nonlinear mean-field dynamo

- Stability: regular or chaotic solutions?

- Intermittency 


\title{
Stable and Unstable Solutions of the Nonlinear Dynamo Problem
}

\author{
Reinhard Meinel \\ Astrophysikalisches Observatorium Potsdam, O-1560 Potsdam, \\ Fed. Rep. of Germany
}

\begin{abstract}
A local potential approach to nonlinear dynamo models which allows the use of variational techniques to investigate the problem of stability is introduced. The method applies at least to quasi-kinematic dynamo models, i.e. to models which include the back-reaction of the magnetic field on the fluid motion in a simplified way. A special application leads to a previously investigated one-dimensional dynamo model which shows a coexistence of a periodic solution (limit cycle) with two stable steady solutions of opposite polarities. The inclusion of some small-amplitude noise leads to interesting transition phenomena which may be of relevance to explain the behaviour of astrophysical dynamos. A simple dynamical system with a two-dimensional phase-space is used for illustration.
\end{abstract}

\section{Introduction}

The kinematic dynamo theory can be regarded as a theory which investigates the stability of hydrodynamic configurations in electrically conducting media with respect to small magnetic fields $B$. In other words, the stability of the state $B \equiv \mathbf{0}$ is analyzed. If this state free of magnetic field proves to be unstable only the full set of coupled magnetohydrodynamic equations can give an answer to the question which state is realized then. Again the problem of stability is important. Only solutions of the magnetohydrodynamic equations which are stable with respect to small (hydrodynamic and magnetic) perturbations are relevant for a description of long-living magnetic configurations in astrophysical objects.

Most of the results presented so far in the field of nonlinear dynamo theory belong to a kind of models which may be called quasi-kinematic mean-field dynamo models. In these models the back-reaction of the magnetic field on the fluid motion is described by more or less well-founded assumptions about the dependence of hydrodynamic functions on the (mean) magnetic field. As kinematic dynamo models these models lead to differential equations for the magnetic field alone, i. e. the velocity field - including its dependence on the magnetic field - is considered 
as given. These models are characterized thus by a disproportion in the degree of physical accuracy: they represent the electrodynamics quite accurately but are rather crude with respect to the hydrodynamics. Nevertheless a lot of interesting bifurcations to steady, periodic, and quasiperiodic solutions have been found for such models (e. g. Brandenburg et al., 1989; Schmitt and Schüssler, 1989; Jennings et al., 1990).

In the present paper we show that all these models can be derived from a simple variational principle. We follow a method of Glansdorff and Prigogine (1971) who developed a variational technique for dissipative systems. A specialization leads back to a one-dimensional dynamo model that was introduced by Krause and Meinel (1988). We compare the results of Meinel and Brandenburg (1990) for this model with the properties of a simple dynamical system. In this way a possible explanation of an irregular time-behaviour of astrophysical dynamos (including the geo-dynamo) becomes more plausible.

\section{A variational principle}

Consider the following Lagrange density depending on two vector functions $B$ and $\boldsymbol{B}_{0}$ :

$$
\mathcal{L}\left(B, B_{0}\right)=\frac{1}{2} \eta(\operatorname{curl} B)^{2}-\left(u \times B_{0}+\mathcal{E}\right) \cdot \operatorname{curl} B+B \cdot \dot{B}_{0}
$$

where the positive-definite scalar function $\eta$ and the vector functions $u$ and $\mathcal{E}$ may depend on $\boldsymbol{B}_{\mathbf{0}}$ and the coordinates explicitly:

$$
\eta=\eta\left(\boldsymbol{x}, \boldsymbol{B}_{\mathbf{0}}\right), \quad \boldsymbol{u}=\boldsymbol{u}\left(\boldsymbol{x}, \boldsymbol{B}_{\mathbf{0}}\right), \quad \mathcal{E}=\mathcal{E}\left(\boldsymbol{x}, \boldsymbol{B}_{\mathbf{0}}\right) .
$$

A dot denotes a time derivative.

Now we calculate the variational derivative of $\mathcal{L}$ with respect to $B$, take it at $\boldsymbol{B}=\boldsymbol{B}_{\mathbf{0}}$, and set it equal to zero:

$$
\left.\frac{\delta \mathcal{L}}{\delta B}\right|_{\mathbf{B}=\mathbf{B}_{\mathbf{0}}}=\mathbf{0}
$$

In components $\delta \mathcal{L} / \delta \boldsymbol{B}$ reads

$$
\left(\frac{\delta \mathcal{L}}{\delta B}\right)_{i}=\frac{\partial \mathcal{L}}{\partial B_{i}}-\left(\frac{\partial \mathcal{L}}{\partial B_{i, j}}\right)_{, j}
$$

where the usual summation convention is used, and $B_{i, j}$ means $\partial B_{i} / \partial x_{j}$.

Equation (3) is equivalent to

$$
\dot{B}_{\mathbf{0}}=\operatorname{curl}\left(u \times B_{0}+\mathcal{E}\right)-\operatorname{curl}\left(\eta \operatorname{curl} B_{\mathbf{0}}\right)
$$

which is just the standard form of the induction equation of mean-field magnetohydrodynamics (cf. Krause and Rädler 1980). $\boldsymbol{B}_{0}$ is the mean magnetic field, $u$ the mean velocity field, $\mathcal{E}$ the mean electromotive force describing the large-scale induction effects of small-scale turbulent motions, and $\eta$ the magnetic diffusivity. 
Together with (2) various types of nonlinear dynamo models are included in this formalism. The so-called $\alpha$-quenching, for instance, is described by

$$
\mathcal{E}=\alpha B_{0}
$$

with an $\alpha$-coefficient depending on $\boldsymbol{B}_{\mathbf{0}}$ such that $\alpha$ vanishes as $\left|\boldsymbol{B}_{\mathbf{0}}\right| \rightarrow \infty$ (Rüdiger, 1974). The effects of magnetic buoyancy, as another example, can be modelled by

$$
u=u_{0}+u_{B}\left(B_{0}\right)
$$

for further details see Moss et al. (1990).

The Lagrange density $\mathcal{L}\left(\boldsymbol{B}, \boldsymbol{B}_{\mathbf{0}}\right)$ defined in (1) can be interpreted as a "local potential" in the sense of Glansdorff and Prigogine (1971). To show this we consider a finite volume $V$ and restrict ourselves, for simplicity, to perfect conductor boundary conditions, i. e. we assume that the tangential components of the electric field $\boldsymbol{E}=\eta \operatorname{curl} \boldsymbol{B}_{\mathbf{0}}-\left(\boldsymbol{u} \times \boldsymbol{B}_{\mathbf{0}}+\mathcal{E}\right)$ vanish at the boundary. We consider the functional

$$
\Phi\left(\boldsymbol{B}, \boldsymbol{B}_{\mathbf{0}}\right)=\int_{V} \mathcal{L}\left(\boldsymbol{B}, \boldsymbol{B}_{\mathbf{0}}\right) d V
$$

and find for $B_{0}$ satisfying (5) and $B \neq B_{0}$

$$
\Delta \Phi \equiv \Phi\left(B, B_{0}\right)-\Phi\left(B_{0}, B_{0}\right)=\frac{1}{2} \int_{V} \eta\left[\operatorname{curl}\left(B-B_{0}\right)\right]^{2} d V>0,
$$

i. e. $\Phi\left(B, B_{0}\right)$ has an absolute minimum at $\boldsymbol{B}=\boldsymbol{B}_{\mathbf{0}}$. This minimum property can be employed to investigate (5) by means of variational techniques, e.g. the Ritz method. In this way a derivation of the Galerkin expansion is also possible which automatically guarantees its convergence (cf. Glansdorff and Prigogine, 1971).

\section{Reduction to a one-dimensional model}

\subsection{The local potential}

Now we assume that the magnetic field depends only on one spatial (cartesian) coordinate, say $z$, and that the component $B_{z}$ vanishes:

$$
\boldsymbol{B}=\left[\begin{array}{lll}
B_{x}(z, t), \quad B_{y}(z, t), & 0
\end{array}\right] .
$$

The components $B_{x}$ and $B_{y}$ can be combined to form the complex function

$$
B \equiv B_{x}+\mathrm{i} B_{y} .
$$

Inserting (10) into (1) we obtain with (6) and $u \equiv 0$

$$
\mathcal{L}\left(B, B_{0}\right)=\frac{1}{2}\left[\eta B^{\prime} B^{* \prime}+\mathrm{i} \alpha\left(B_{0} B^{* \prime}-B_{0}^{*} B^{\prime}\right)+B \dot{B}_{0}^{*}+B^{*} \dot{B}_{0}\right],
$$

where an asterisk denotes complex conjugation and a prime denotes differentiation with respect to $z$. Assuming 


$$
\eta=\text { const }>0, \quad \alpha=\alpha_{0} f\left(B_{0}^{*} B_{0}\right), \quad \alpha_{0}=\text { const }>0,
$$

and introducing dimensionless space- and time-coordinates we find

$$
\mathcal{L}\left(B, B_{0}\right)=B^{\prime} B^{* \prime}+\mathrm{i} C f\left(B_{0}^{*} B_{0}\right)\left(B_{0} B^{* \prime}-B_{0}^{*} B^{\prime}\right)+B \dot{B}_{0}^{*}+B^{*} \dot{B}_{0} .
$$

(We have omitted the unnecessary factor $\frac{1}{2}$.) The dimensionless "dynamo number" $C$ is defined by

$$
C=\frac{\alpha_{0} d}{\eta}
$$

where $d$ is the thickness of the electrically conducting slab under consideration.

Equation (3) reads now

$$
\left.\frac{\delta \mathcal{L}}{\delta B^{*}}\right|_{B=B_{0}}=0
$$

with

$$
\frac{\delta \mathcal{L}}{\delta B^{*}}=\frac{\partial \mathcal{L}}{\partial B^{*}}-\left(\frac{\partial \mathcal{L}}{\partial B^{* 1}}\right)^{\prime}
$$

and leads to

$$
\dot{B}_{0}=B_{0}^{\prime \prime}+\mathrm{i} C\left[f\left(B_{0}^{*} B_{0}\right) B_{0}\right]^{\prime} .
$$

(Note that the variational derivative of $\mathcal{L}$ with respect to $B$ gives the complex conjugate of (18).)

We assume that $B_{0}$ and $B$ vanish at the boundaries $z=0$ and $z=1$ of the slab:

$$
\begin{gathered}
B_{0}(0, t)=B_{0}(1, t)=0, \\
B(0, t)=B(1, t)=0 .
\end{gathered}
$$

These boundary conditions correspond to a vacuum (free of magnetic field) for $|z|>1$.

Now we consider the functional

$$
\Phi\left(B, B_{0}\right)=\int_{0}^{1} \mathcal{L}\left(B, B_{0}\right) d z
$$

and calculate

$$
\Delta \Phi \equiv \Phi\left(B, B_{0}\right)-\Phi\left(B_{0}, B_{0}\right)
$$

for $B \neq B_{0}$. With (18), (19) and (20) we obtain by integration by parts

$$
\Delta \Phi=\int_{0}^{1}\left(B^{\prime}-B_{0}^{\prime}\right)\left(B^{\prime}-B_{0}^{\prime}\right)^{*} d z>0 .
$$

Thus $\Phi$ is again a "local potential". The one-dimensional nonlinear dynamo model (18), (19) was investigated by Krause and Meinel (1988) and Meinel and Brandenburg (1990). 


\subsection{Stable and unstable solutions}

Various solutions of (18), (19) have been obtained for the following assumptions about the function $f$ :

$$
\begin{gathered}
f=1-B^{*} B, \\
f=\frac{1}{\left(1+B^{*} B\right)^{2}}, \\
f=\frac{1}{1+\left(B^{*} B\right)^{\kappa}}, \quad \kappa \geq 1 .
\end{gathered}
$$

At first, for $C>2 \pi$, there is always a stable steady solution $B_{\mathbf{s}}$. It bifurcates from the solution $B \equiv 0$ at $C=C_{\text {crit }}=2 \pi$. At $C=2 n \pi, n=2,3,4, \ldots$, unstable steady solutions bifurcate from $B \equiv 0$. All steady solutions can be found analytically up to a single real quadrature. In case (24) the steady solutions can even be given explicitly in terms of elliptic functions.

It should be noted that, if $B$ is any solution of (18), (19), then $B \exp \left(\mathrm{i} \varphi_{0}\right)$ also satisfies (18), (19), where $\varphi_{0}$ is a real constant. We restrict this gauge freedom by the constraint

$$
B^{*}(1-z, t)=B(z, t),
$$

which means that the real part of $B$ is an even function and the imaginary part of $B$ an odd function of $z$ with respect to the midpoint $z=\frac{1}{2}$ of the considered interval $[0,1]$. Note that, according to (18), (19), the constraint (27) is conserved in course of time if it is initially satisfied. This constraint, however, leaves the freedom $B \rightarrow-B$, i. e. for a given solution $B,-B$ is also a solution of our problem. Thus, for $C>2 \pi$, we have exactly two stable steady solutions $B_{\mathbf{s}}$ and $-B_{\mathbf{s}}$. Which of them is attained as the final state of evolution depends on the initial conditions. Numerical investigations of (18), (19) revealed that for large values of the dynamo number $C$ there is also a third possibility for the final state of evolution: a stable oscillating solution $B_{\mathrm{osc}}$. The basin of attraction of this limit cycle increases with growing $C$ while the basins of the two steady solutions shrink. The oscillating solution changes periodically its sign:

$$
B_{\text {osc }}(z, t+T)=-B_{\text {osc }}(z, t) .
$$

The full cycle period is given by $2 T$.

\subsection{Noise-induced transitions}

For large values of $\kappa$ in case (26) the oscillating solution $B_{\text {osc }}$, at particular time instants $t=t_{\mathrm{e}}$ (turning points), closely approaches the steady solutions $\pm B_{\mathbf{s}}$. This approach is the closer the larger the value of $\kappa$. A close approach means that

$$
\int_{0}^{1}\left|B_{\mathrm{osc}}(z, t) \mp B_{\mathrm{s}}\right|^{2} d z
$$

is a small quantity for $t=t_{\mathrm{e}}$. Since both solutions $B_{\mathrm{s}}$ and $B_{\mathrm{osc}}$ are stable with respect to infinitesimal perturbations there exists a basin boundary between $B_{\mathrm{s}}$ and 
$B_{\text {osc }}\left(t_{\mathrm{e}}\right)$. However, it is clear that a suitable small finite perturbation is sufficient to produce a jump from $B_{\mathrm{s}}$ to $B_{\mathrm{osc}}$ and vice versa. Therefore the inclusion of a small noise-term to (18) may lead to an interesting time-behaviour of the resulting solutions. Figure 1 shows solutions of

$$
\dot{B}=B^{\prime \prime}+\mathrm{i} C\left[f\left(B^{*} B\right) B\right]^{\prime}+A G(z) F(t)
$$

with $G(x)=\sin \pi z+\frac{\mathrm{i}}{2} \sin 2 \pi z$ and $F$ is a stochastic function:

$$
F(t)=\sum_{k=1}^{\infty} \epsilon_{k} \delta(t-k \Delta t), \quad t>0
$$

where $\epsilon_{k}$, for each $k$, can take the values $-1,0,1$ with probabilities $p, 1-2 p, p$, respectively. $A=0.2, p=10^{-2}, \Delta t=10^{-3}$. The function $f$ was chosen according to $(26)$ and $\kappa=4$. The steady solutions $\pm B_{\mathrm{s}}$ are characterized by $\operatorname{Re} B\left(z=\frac{1}{2}\right) \approx$ \pm 1 . Obviously, the noise is sufficient for $B(z, t)$ to leave the basin of attraction of $B_{\mathrm{s}}$. The system follows then a part of $B_{\mathrm{osc}}$. In this way it is even possible to reach $-B_{\mathrm{s}}$, i. e. to generate a reversal of the magnetic field. With increasing $C$ the oscillating solution and with decreasing $C$ the steady solutions dominate. (Note that $B_{\text {osc }}$ exists as a stable solution for $C \gtrless 48$ only.)

In general, the noise-level which is necessary to produce jumps between the different attractors depends on the dynamo number $C$ and on the degree of the nonlinearity $f$, e.g. on $\kappa$.

\subsection{An illustrative example with a two-dimensional phase-space}

The system (18), (19) has an infinite-dimensional phase-space. A discussion of basin boundaries etc. is therefore very difficult. The aim of this section is to present a "toy" system which shows the same coexistence of attractors as (18), (19) but is as simple as possible. A dynamical system of the form

$$
\begin{gathered}
\dot{x}=F(x, y), \\
\dot{y}=G(x, y)
\end{gathered}
$$

is sufficient for this purpose. We choose

$$
\begin{aligned}
& F(x, y)=x J(x, y)-y K(x, y) \\
& G(x, y)=y J(x, y)+x K(x, y)
\end{aligned}
$$

with

$$
\begin{gathered}
J(x, y)=A-B\left(x^{2}+y^{2}\right)+C\left(x^{2}+y^{2}\right)^{2}-\left(x^{2}+y^{2}\right)^{3} \\
K(x, y)=2-\left(x^{2}+y^{2}\right)-\frac{2}{5} x y
\end{gathered}
$$

and take

$$
A=15, \quad B=18.5, \quad C=7.5 \text {. }
$$

This system has the following attractors: 

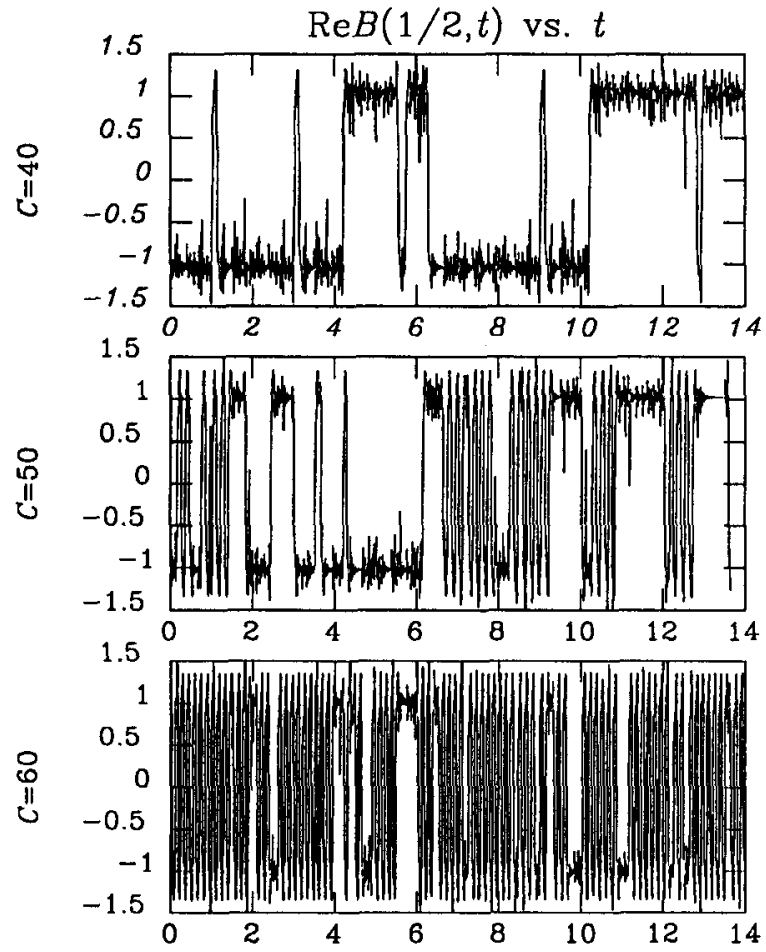

Fig. 1. Time-behaviour of the dynamo model with coexisting two steady and one periodic attractors under the influence of a small-amplitude noise for different values of the dynamo number $C$ (Meinel and Brandenburg, 1990)

$$
\begin{gathered}
\text { (i) } x= \pm \sqrt{2}, \quad y=0, \\
\text { (ii) } x^{2}+y^{2}=3
\end{gathered}
$$

i.e. two fixed points and one limit cycle. Unstable steady solutions are given by $x=y=0 ; x=0, y= \pm \sqrt{2} ; x=-y= \pm \sqrt{5 / 4}$. The two-dimensional phasespace is shown in Fig. 2. It can be seen that the limit cycle, when crossing the $x$-axis, closely approaches the fixed points. The basin boundary of the limit cycle is given by the circle $x^{2}+y^{2}=\frac{5}{2}$. All trajectories starting at $x^{2}+y^{2}>\frac{5}{2}$ reach asymptotically the limit cycle. All trajectories starting at $x^{2}+y^{2}<\frac{5}{2}$ reach one of the fixed points. The boundary between the basins of the two fixed points can also be seen from Fig. 2 approximately.

The behaviour of our system (32), (33) can easily be understood by introducing polar coordinates:

$$
x=r \cos \varphi, \quad y=r \sin \varphi .
$$

Together with (34)-(38) this leads to 


$$
\begin{gathered}
\dot{r}=r\left(2-r^{2}\right)\left(\frac{5}{2}-r^{2}\right)\left(3-r^{2}\right), \\
\dot{\varphi}=2-r^{2}-\frac{1}{3} r^{2} \sin 2 \varphi .
\end{gathered}
$$

The steady solutions (except $x=y=0$ ) are simply given by the crossing points of the isoclinals $\dot{r}=0$ (circles) and $\dot{\varphi}=0$ (ellipse). Note that $\dot{\varphi}<0$ for all $\varphi$ on the circle $r^{2}=3$ (limit cycle). The basin-boundary property of the circle $r^{2}=\frac{5}{2}$ can easily be deduced from (42): for $r$ slightly exceeding $\sqrt{5 / 2}$ we find $\dot{r}>0$ while $\dot{r}<0$ for $r$ slightly less than $\sqrt{5 / 2}$. In a similar way the stability properties of the steady solutions and the limit cycle can be found.

Looking at Fig. 2 one can easily imagine a time-behaviour completely comparable to that of Fig. 1 if some small noise is added to (32), (33).

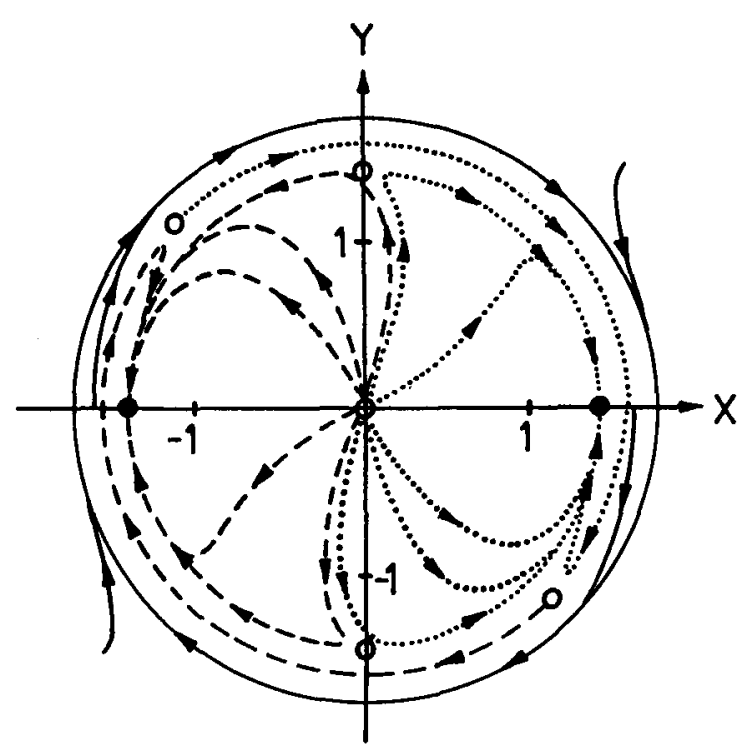

Fig. 2. The trajectories for the "toy" system lead either to the limit cycle $x^{2}+y^{2}=3$ (full lines) or to one of the two fixed points $x= \pm \sqrt{2}, y=0$ (dotted resp. dashed lines). Small empty circles indicate unstable steady solutions

\section{Discussion}

The coexistence of two stable steady solutions (which differ only in their polarity) and a periodic solution which closely approaches these steady solutions offers an interesting possibility for explaining the irregular time-behaviour of the magnetic field of various astrophysical objects. (Stability means here only stability with 
respect to infinitesimal perturbations - but not with respect to small finite perturbations.) The assumption of some small noise is quite natural. It is even not necessary to make use of external perturbations to explain it. Within the frame of mean-field theory a small noise term may occur as a correction to the induction equation (5) if the Reynolds relations are not exactly satisfied in the usual two-scale approach (cf. Hoyng, 1988).

Such an explanation of a random-like behaviour of mean-field dynamo models seems to be at least as plausible as an explanation based on deterministic chaos.

In particular, the behaviour of the Earth's magnetic field as obtained from paleomagnetic records can be compared with our solutions, e.g. in the upper panel of Fig. 1. The lower panel of Fig. 1 which corresponds to a larger value of the dynamo number, where the periodic solution dominates, may be of interest for explaining the activity behaviour observed for young and very active stars. Also the solar cycle is not exactly periodic.

Of course, the nonlinear dynamo models under consideration are far away from real astrophysical objects. This is, however, just an argument in favour of simple models. It does not make much sense to study too complicated models of this "quasi-kinematic" kind (three-dimensional models with many free parameters etc.). To be closer to reality the hydrodynamic part of the theory must be treated more thoroughly.

The variational principle discussed in section 2 can certainly be generalized to the full magnetohydrodynamic equations.

\section{References}

Brandenburg, A., Krause, F., Meinel, R., Moss, D., Tuominen, I.: 1989, Astron. Astrophys. 213, 411

Glansdorff, P., Prigogine, I.: 1971, Thermodynamic theory of structure, stability and fluctuations, Wiley-Interscience, London

Hoyng, P.: 1988, Astrophys. J. 332, 857

Jennings, R., Brandenburg, A., Moss, D., Tuominen, I.: 1990, Astron. Astrophys. 230, 463

Krause, F., Meinel, R.: 1988, Geophys. Astrophys. Fluid Dyn. 43, 95

Krause, F., Rädler, K.-H.: 1980, Mean-field magnetohydrodynamics and dynamo theory, Akademie-Verlag, Berlin

Meinel, R., Brandenburg, A.: 1990, Astron. Astrophys. 238, 369

Moss, D., Tuominen, I., Brandenburg, A.: 1990, Astron. Astrophys. 228, 284

Rüdiger, G.: 1974, Astron. Nachr. 295, 275

Schmitt, D., Schüssler, M.: 1989, Astron. Astrophys. 223, 343 